\title{
An REU Experience With Micro Assembly Workcell Research
}

William Stapleton, Texas State University - San Marcos, USA

Bahram Asiabanpour, Texas State University - San Marcos, USA Jesus Jimenez, Texas State University - San Marcos, USA Dugan Um, Texas A\&M University - Corpus Christi, USA

\begin{abstract}
Under an NSF REU center grant REU-0755355 entitled "Micro/Nano Assembly Workcell Via Micro Visual Sensing and Haptic Feedback", Texas A\&M University - Corpus Christi and Texas State University - San Marcos collaboratively hosted two groups of 10 students from different backgrounds for 10 weeks each in Summer 2008 and 2009 respectively. The research effort involved is part of an ongoing research program developing novel methods for making automated micromanipulation systems. The twenty students and two teachers were divided into four teams developing interrelated aspects of the project. The first team developed the micro-scale parts, such as gears, for assembly. This required the team to develop techniques for etch and release of three dimensional parts from a silicon substrate. The second team developed the microscopic vision system used for locating and identifying parts on a silicon wafer. The vision system is used to determine the position on the wafer of the parts, their diameter (one of several standard parts), and, utilizing a novel technique developed by the team, the thickness of the parts. The third team developed a robotic platform able to locate any portion of a wafer for manipulation within a three-dimensional space with $10 \mu \mathrm{m}$ accuracy. This mechanism is used to first bring any desired portion of the wafer to the vision system for analysis and also to bring a manipulator to "pick and place" parts using the vision system for feedback. The fourth group developed the micromanipulator in the form of a "gripper" powered by electro-active polymer. This gripper was capable of accurately and repeatably gripping, lifting, moving, placing, and releasing parts at any point within the workspace. Collectively, the REU project successfully produced a prototype system advancing the state-of-art for an important are of micro-manufacturing while offering a stimulating experience for undergraduate students. Assessment of the student experience showed an increase in the likelihood for these students to pursue engineering careers and to encourage their peers to consider STEM careers.
\end{abstract}

Keywords: REU; computer vision; micro-manufacturing

\section{INTRODUCTION}

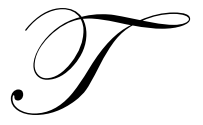

he objective of the NSF REU (Research Experience for Undergraduates) Site "Micro/nano Assembly Workcell via Micro Visual Sensing" (REU-0755355) was to provide hands-on training in micromanufacturing research to a total of 20 student participants over a two-year period from June 2008 to August 2009. This is part of an on-going research effort to develop prototypes of a micro assembly system that can handle components smaller than $500 \mu \mathrm{m}$. The student participants were selected from national institutions where research facilities in this field are limited. A special emphasis was placed on recruiting women and historically underrepresented minorities. Of the students participating in the program, $40 \%$ were female. The ethnic breakdown self-reported by the students was 55\% white, 30\% Hispanic, 10\% African-American, and 5\% other.

Student participants contributed to the development of a new micro-manufacturing system as a common ground of the research. The students, who uniformly reported a positive experience via a series of surveys, will be more likely to remain in the technical fields after having hands-on research experiences. Their rewarding 
experiences will naturally encourage further participation with future research endeavors.

The micro-manufacturing system developed by the students consists generally of a microgripper system in conjunction with a vision system intended for "pick and place" operations with MEMS parts. From the science and technology standpoint, successful development of micro-grippers and a 3D micro positioning system opens endless application opportunities in homeland security, transportation, aerospace, biomedical, advanced manufacturing, and many other commercial applications such as hearing aids or minimally invasive surgical tools. Micro-manufacturing systems have been developed primarily to assemble a matrix of arranged micro components for various microstructures. Novel processes like LIGA (German acronym for lithography, electroplating, and molding), microcasting, and micro electrical discharge machining (microEDM) have been developed to fabricate microcomponents for miniature machines. On the manufacturing side of such miniaturized components, however, no efficient or automated assembly system has been invented yet. The research aspect of this REU aims to develop a novel microassembly system that can "see" and "feel" when handling micro objects. Development of microdevices, micro sensors, and microactuators has been escalating due to increasing trends for product miniaturization and advances in science and technology. The market for such microsystems has been climbing at a rate of $20 \%$ to $30 \%$ per year since 1990; the worldwide market for microsystems has been estimated to approach 30 billion dollars in 2009 [1]. Full blown application of microsystems, however, is hindered by manufacturing constraints together with a high assembly cost. A comprehensive study showed the assembly cost is more than half of the microdevice cost [2].

Ideal microgrippers would be able to handle a variety of micro/nano components for biological, medical, aerospace, manufacturing, chemical, or security applications. Most commercially available devices are open loop system, i.e., a trained operator is required to operate the system at high magnification. Without sensitive feedback, handling of fragile biological cells and advanced materials such as carbon nanotubes or optical fibers would be difficult since an operator has to constantly watch for minute shape change of the sample or risk damaging it. Although microassembly of prototypes and low volume microsystems could be done with skillful operators, having human operators assembling high volume microsystems would not be economically justifiable due to high labor and training cost, fatigue, low yield, and inconsistent product quality. The risk is even higher when having a human operator working on hazardous chemicals, radioactive components, or contaminated biological microtissues.

While some preexisting micro-manufacturing systems such as one from Zyvex, Inc. provide excellent accuracy, they lack the flexibility to span the assembly system to the next level of technical domain, including military, homeland security, transportation, aerospace, and other commercial applications due to the material constraints. Only semiconductor material fabricated on a silicon substrate can be assembled. The micro gripper system developed by Sandia National Laboratory demonstrates excellent object identification and manipulation capability. The micro-manufacturing cell composed of several compartments can cope with up to $100 \mu \mathrm{m}$ parts with its multi-camera micro vision system. Since the image processing system, however, works only off-line, the application area is significantly limited. Furthermore, next generation micro-manufacturing systems are required to be flexible enough to be able to handle various materials in arbitrary shapes with tactile feedback. To address these needs, research is being conducted to develop novel micro-manufacturing systems that are autonomous, intelligent, and flexible in structure. Such micro-manufacturing systems can assemble or disassemble arbitrary components based on assembly sequence maps to deal with mission critical parts such as micro sensors, micro actuators or biologically/chemically hazardous embodiments, etc.

\section{THE REU RESEARCH}

A micro-manufacturing system is well suited to an REU Site project because potential applications are exciting and research topics encompass a variety of disciplines, making the research naturally interdisciplinary. Key areas of research in which students can participate include mechanics, micro sensor/actuator design, electronics/control system design, design optimization, and advanced micro-manufacturing. The integration of such research activity will result in development of new technologies for automatic microassembly systems. A sensitive yet economical force sensor integrated with a micro-gripper would be useful to quantitatively measure the applied force on the actuator. Such a "touch sensor" prevents damage to critical and expensive samples and is utilized for instant haptic feedback. A vision system identifies and calculates the precise location and orientation of a 
microcomponent before manipulating it to the intended position. Such an "eye sensor" provides the feedback to a microrobotic system for efficient microassembly. The autonomous microassembly system would free human operators for more intelligent and less tedious tasks and simultaneously improve quality and reduce cost of the microproducts. The success of the project is demonstrated with (i) development of a microscopic infrared 3D vision system for micro parts identification with image recognition and the capability to obtain position and orientation data, (ii) development of a microgripper with sensitive haptic feedback and a multi-axis motion system, and (iii) integration of the two systems for autonomous microassembly. This project provides intelligence and autonomy to the improved microgrippers with haptic and vision feedback. To make the project realistically manageable within two years of REU Site research, the scope of this project has been simplified without changing the project objectives: (i) Simplifying the component to a simple micro object and performing the two dimensional rather than a three dimensional microassembly task, (ii) Enhancing the technology, an infrared proximity array, into an intelligent microscopic visual feedback system for micron/sub-micron components, (iii) integration of both vision and microgripping systems for an autonomous and closed- loop microassembly system.

The students participating in the REU were organized into four groups tasked with different portions of the overall project. The four groups were the "Robotics" group, the "MEMS" group, the "3-D Vision" group, and the "Micro Gripper" group. All of the groups met together for instructional sessions in which technical background information and supplemental instruction were provided. Each group also received separate instruction specific to their task. The groups were guided to interact closely to ensure that their individual work products could be integrated into a complete system.

The task for the MEMS group was to create a number of micro gears and similar items of interest for later manipulation by the microgripper system. These parts were fabricated from silicon wafers using a bulk micromachining process. The students prepared a design mask which appears in Figure 1. After considerable experimentation, the students refined a development process that allowed for a suitable etch of these parts which avoided significant under-etch or over-etch. The resulting "good" gears were used by the other groups as necessary.

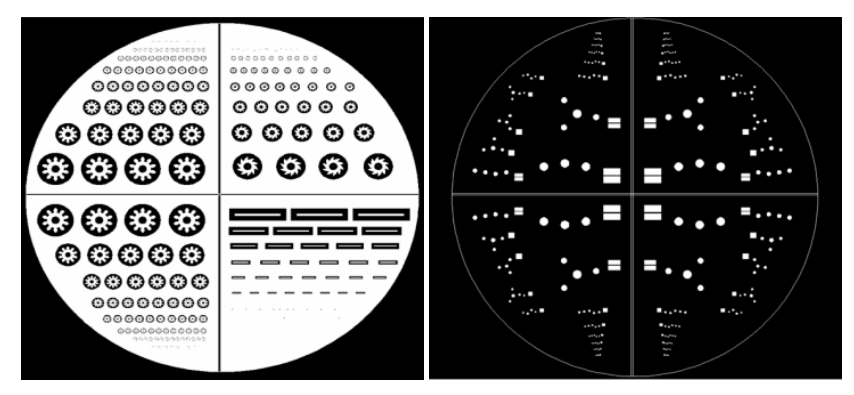

Figure 1: The Micro Gear Mask

The task for the 3-D vision group was to create a system which utilized the properties of a standard infrared (IR) video sensor to generate a perception of "pseudo-depth" using a single sensor. The microscope camera system is shown in Figure 2. Initially, the approach was to set an emitter of IR light to illuminate the portion of the work area under examination and measure the amount of the reflected light received for each pixel [5]. Given the assumption that IR light will be absorbed proportionally with distance from light source to object, the distance from the sensor to any point on the object could be estimated with sufficient resolution to clearly distinguish the nominal $40 \mu \mathrm{m}$ vertical edges of the sample gears and other items created as test items for the microgripper [6]. With direct lighting, this "pseudo-depth" apparatus was able to produce quite useful images such as those in Figure 3. Unfortunately, the method proved extremely sensitive to the angle of incident light with non-direct light causing false edges to be identified. Consequently, the team created a light collar for the microscope to produce a bright, even light. 


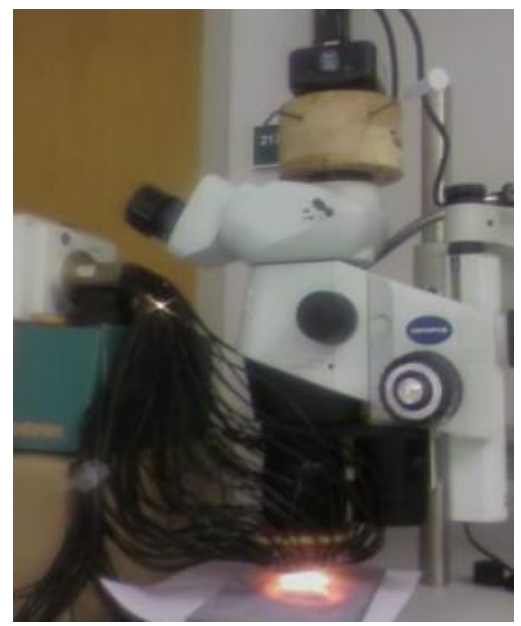

Figure 2: The 3-D Vision System
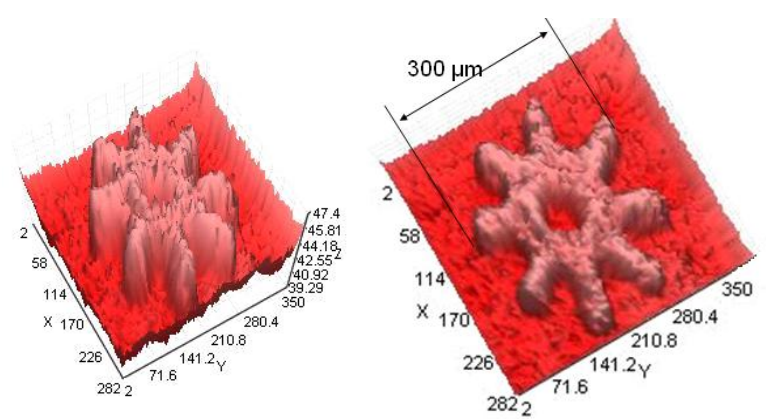

Figure 3: Pseudo-depth IR Image of Micro Gear generated by 3-D Vision Team

The task of the Micro Gripper group was to develop a mechanism which could be used to manipulate the micro gears developed by the MEMS group and located by the 3-D Vision group. The decision was made to use Electro-active Polymer (EAP) as the basis for the gripper [3]. Applying an electric voltage to EAP causes a physical deformation of the material which is reversible by removing the applied voltage. This allows for precisely controlled movement on micro and nano scales which is reliably repeatable over the lifetime of the material. Figure 4 shows a sample of the EAP material in both its inert and energized states.

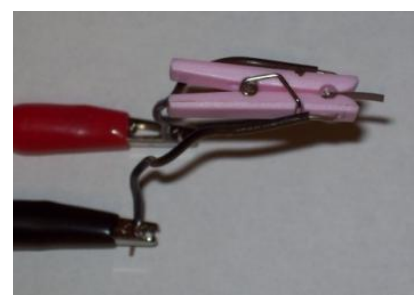

(a) No voltage applied

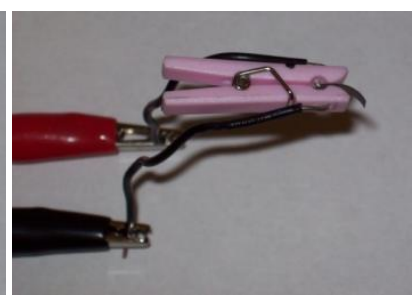

(b) Voltage applied

Figure 4: Deformation of EAP Material with Applied Voltage

Utilizing this property of the EAP as a source of motion, a gripper was designed as shown in Figure 5. Requirements for the gripper include a small enough contact point to be able to grip a single object without interfering with other objects [4]. The gripper arms must have a very low profile off of the surface of the work area in order to pass underneath the lens of the vision system. The length of the gripper arms must be sufficient to reach any point on the work surface. 


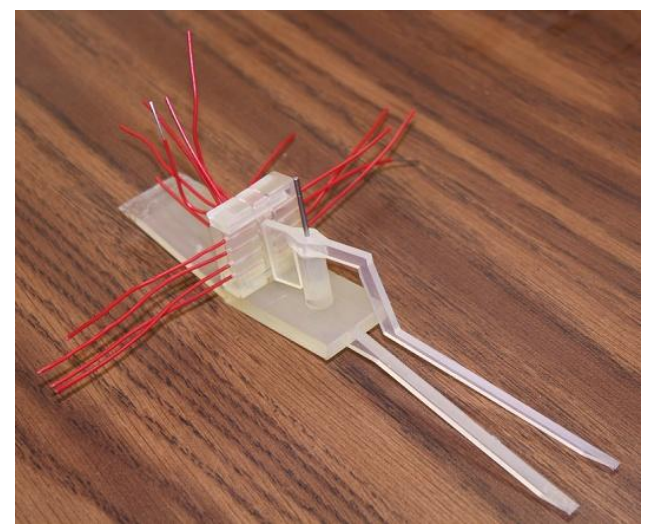

Figure 5: The EAP-powered Gripper

The task of the Robotics group was to build a three-degree-of-freedom platform which would allow the micro gripper to be precisely placed at a set of three-dimensional coordinates supplied by the 3-D vision system. Figure 6 shows the robotic platform with the micro gripper in place. This group designed the mechanical linkages to position the wafer serving a platform for the parts and the platform to hold the parts. This group also produced the software necessary to control the placement of the gripper based on the coordinates of the object to be manipulated.

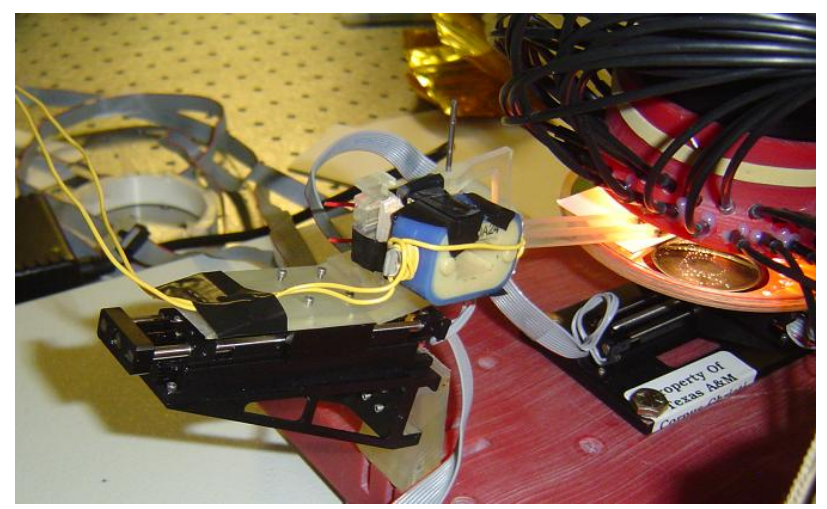

Figure 6: Robotic Arm with Holder and EAP Micro-grippers

Once all of the groups performed their individual tasks, the various components were assembled into the complete system shown in Figure 6. Under manual control, the gripper was able to lift and place parts. Unfortunately, by the end of the second 10-week session, fully automated part selection and placement was not achieved. However, the components of the system are in place. Future efforts should be able to build on this framework to complete a fully automated platform.

\section{STUDENT ASSESSMENT}

Assessing the performance of the students involved in the REU in terms of the technical aspects of their research is the purview of the faculty members leading the REU rather than the students. Of equal importance to the outcome of the REU is the effect the experience will have on the eventual careers of the participants. This aspect is most directly measured by the students themselves. The students were administered a series of carefully crafted survey instruments used to assess the students' perspectives on how the experience is shaping their careers. The first set of instruments used for this student self-assessment involved a matched pair of pre-experience and postexperience surveys. 
On the pre- and post-experience surveys, when asked to self-assess their "future plan to pursue a career involving research," the students began the process with an average score of 6.9/10 and ended the process with an average score of 8.1/10 indicating a distinctly increased interest in STEM (science, technology, engineering, and mathematics) related careers. Furthermore, every student responded at the same level or higher on the postexperience survey. No student's interest in a STEM-related career was negatively impacted. The number of participating students is too small for further statistical analysis to be conclusive.

On the post-experience survey, the students' response to "How did the "hands-on" learning activities using the provided equipment influence your learning and research?" was an average of 8.2/10 indicating that they found the research experience very useful. Similarly, in response to "In general, how would you describe your motivation in this research program?" the students averaged 9.0/10 indicating an excellent motivational level as a result of the REU. The question "Do you think the knowledge and skills you gained in this research program will be useful in your future research or career?" resulted in an average response of 9.2/10.

\section{FUTURE DIRECTIONS}

At the conclusion of the REU experience, the teams of students over the two ten-week session had produced a system with many of the necessary components of a fully automated micromanipulator system. The resulting system could best be described as a guided semi-autonomous system. The authors are seeking funding to continue this project with a future REU experience. As planned, that experience would focus on completing the automation of the micromanipulation system. Under consideration is a redesign of the grippers to allow for manipulation of smaller parts. Similarly, redesigning the camera system to provide higher resolution would allow for resolution of smaller parts.

\section{CONCLUSIONS}

This REU brought together two diverse group of ten students each over two ten-week sessions to confront a significant research challenge in the form of an automated micromanipulation system. Such a system could serve as a prototype for MEMS manufacturing systems. The participants were able to meet most of the challenges set for them completely and were able to make significant progress on all goals. More importantly, all of the participants in the REU indicated a strong increase in their interest in pursuing STEM careers as a result of the experience. By this measure, the REU was successful. A clear plan exists for how to extend the research begun with the REU. Funding for a new REU building on the results from this REU have been solicited. However, regardless of the status of that request, this research will continue to be pursued.

\section{AUTHOR INFORMATION}

William Stapleton, Assistant Professor, Electrical Engineering program, Ingram School of Engineering, Texas State University - San Marcos, wstapleton@ txstate.edu.

Bahram Asiabanpour, Associate Professor, Manufacturing Engineering program, Ingram School of Engineering, Texas State University - San Marcos, ba13@txstate.edu.

Jesus Jimenez, Assistant Professor, Industrial Engineering program, Ingram School of Engineering, Texas State University - San Marcos, Jesus.Jimenez@txstate.edu.

Dugan Um, Assistant Professor, Mechanical Engineering and Engineering Technology, Texas A\&M University Corpus Christi, dugan.um@tamucc.edu.

\section{REFERENCES}

1. McWilliams, A., http://www.bccresearch.com/report/ treport.php?rcode=IAS027A\&type=high, Tech Report ID: IAS027A, July, 2008 
2. $\quad$ Chollet, S.K., Bourgeois, F., and Jacot, J., "Economical Justification of Flexible Microassembly Cells," Proceedings, IEEE Symposium on Assembly and Task Planning, 2003, pp. 48-53.

3. $\quad$ Kornbluh, R., Pelrine, R., Pei, Q., Shastri, S., "Application of Dielectric Elastomer EAP Actuators," Electroactive Polymer (EAP) Actuators as Artificial Muscles, SPIE Press, pp. 457-495, 2001.

4. "MEMS Technology: Where To?," Business report RGB-270, Business Communications Company, Inc., 25 Van Zant Street, Norwalk, CT 06855, 2002.

5. Novotny, P.M. and Ferrier, N.J., "Using Infrared Sensors and the Phong Illumination Model to Measure Distance," IEEE Int. Conf. On Robotics and Automation, pp. 1644-1649, 1999.

6. Um, D. and Hung, W.N.P., "A Novel Infrared Proximity Array Sensor for Micro-Workcell: Modeling and Applications,” IEEE Int. Conf. On Robotics, Automation and Mechatronics, 2006. 
NOTES 